\title{
Matching of the ECRH Transmission Line of W7-X
}

Georg Michel ${ }^{\mathrm{a}}$, Volker Erckmann ${ }^{\mathrm{a}}$, Frank Hollmann ${ }^{\mathrm{a}}$, Lothar Jonitz ${ }^{\mathrm{a}}$, Walter Kasparek ${ }^{\mathrm{b}}$, Heinrich Laqua $^{\mathrm{a}}$, Carsten Lechte $^{\mathrm{b}}$, Nikolai Marushchenko ${ }^{a}$, Burkhard Plaum ${ }^{b}$, Yuriy Turkin ${ }^{\mathrm{a}}$, Michael Weißgerber ${ }^{\mathrm{c}}$

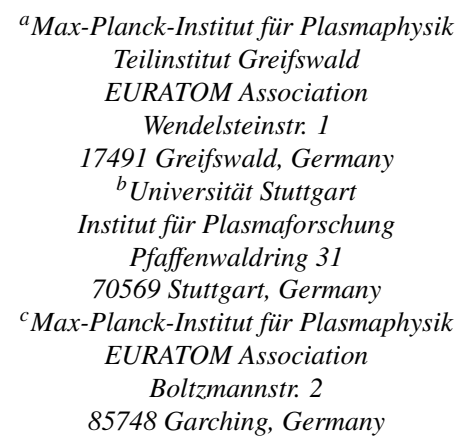

\begin{abstract}
The polarization of the directed ECRH power has to be matched to the plasma boundary with respect to the magnetic field at the density gradient region close to the last closed flux surface (LCFS). This is achieved by means of grooved mirrors, which provide the required polarization and which are part of the matching optics unit (MOU) of the gyrotrons. The RF radiation from the gyrotrons has to pass about 16 mirrors in a complex three-dimensional arrangement in order to reach the plasma. The paper discusses the modeling of the ECRH transmission in order to find the required polarizer adjustment for each possible injection angle and plasma wave type (O- or X-mode). This includes the calculation of the polarization state on the plasma boundary, the back-propagation through the transmission line up to the MOU and finally the calculation of the corresponding angles of both polarizers.
\end{abstract}

Keywords: ECRH, W7-X, Transmission Line

\section{Introduction}

Electron Cyclotron Resonance Heating (ECRH) is the main 25 heating method of the W7-X stellarator in Greifswald, Ger- 26 many. The ECRH plant consists of ten $1 \mathrm{MW} / \mathrm{CW}$ gyrotrons 27 at a frequency of $140 \mathrm{GHz}$ which corresponds to the 2nd har- 28 monic of the W7-X field. For a more detailed description, the 29 reader is referred to [1].

$\mathrm{O}$ - and X-mode are the two plasma waves which exist at ${ }_{31}$ an arbitrarily low density (plasma edge) and which couple to ${ }_{32}$ a TEM wave in vacuum. The purpose of the polarizers close ${ }_{33}$ to the gyrotron is to provide a TEM wave with the right orien- 34 tation, ellipticity and rotation sense in order to couple to the aforementioned plasma waves. The correct matching of the ECRH beams to the plasma is crucial, not only for the maximum heating efficiency and precise localization, but also for the minimization of the RF stray radiation in the plasma vessel. All in-vessel components are exposed to the non-absorbed RF power and have to cope with it. Therefore, all critical components in $\mathrm{W} 7-\mathrm{X}$ are tested against the expected stray radiation level in the MISTRAL test facility [2].

In the following, the ECRH transmission line will be introduced and the modeling of the wave polarization from the gyrotron to the plasma (and back) will be discussed.

\section{The W7-X ECRH Transmission Line}

The ECRH transmission line of the W7-X stellarator has a purely quasi-optical design. It consists of several single- beam waveguides and two multi-beam waveguides for 10 gyrotrons (140GHz, 1MW, CW each). Figure 2 shows an overview over the ECRH plant. The output beams of five gyrotrons are combined into two multi-beams respectively. The multi-beams are transmitted into the torus hall by two multi-beam waveguides (MBWG). At the output, these MBWG's produce an exact image of their input pattern. In this way the individual beams can be separated again in the torus hall.

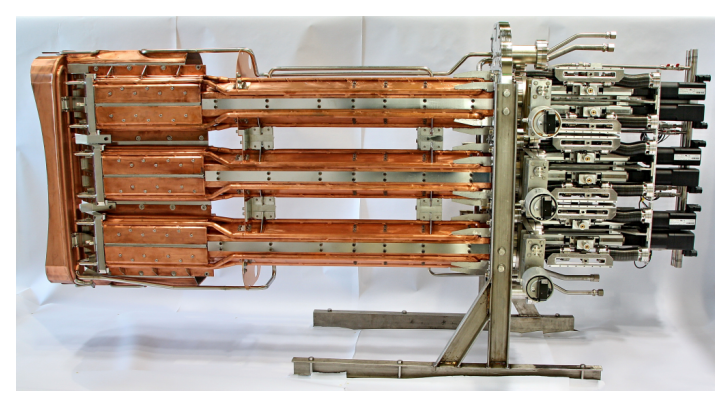

Figure 1: Front Steering Launcher 


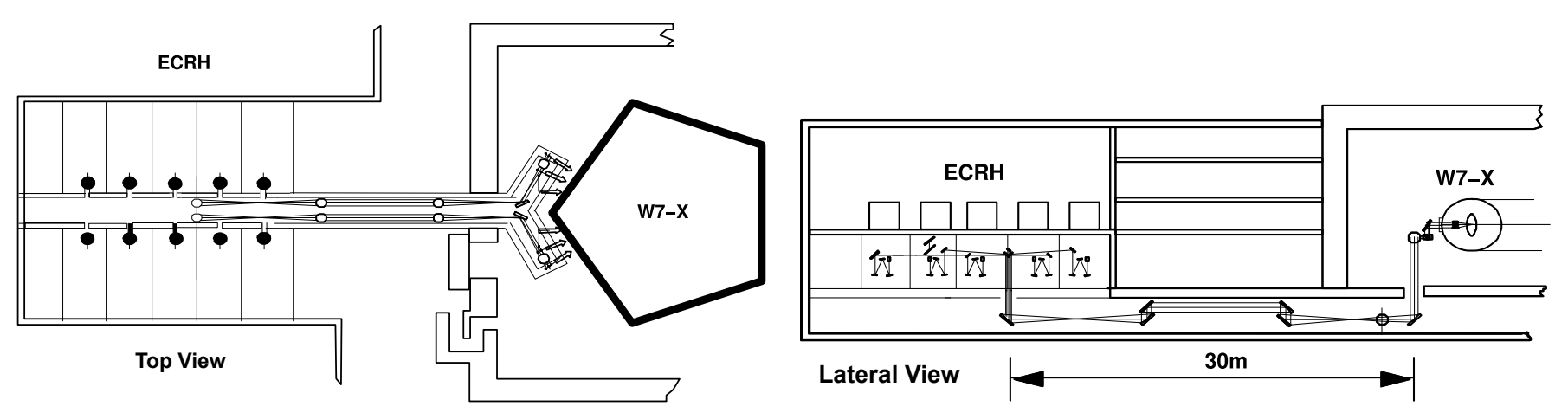

Figure 2: Sketch of the ECRH Plant

The imaging needs to be very exact in order to avoid spillover and mismatching losses. This is achieved by a mirror arrangement of two Gaussian telescopes. Spurious modes are introduced due to the tilt angle of the mirrors in the first telescope. The second telescope transforms these modes back the original mode mix due to symmetry properties.

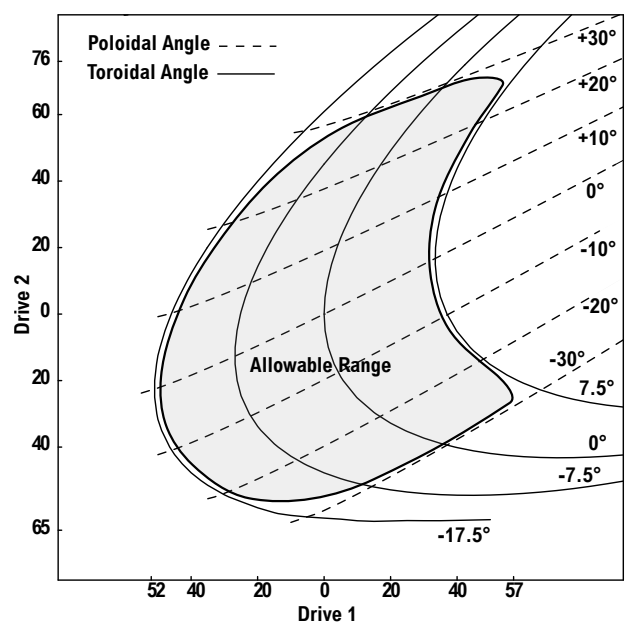

Figure 3: Mapping Function of the Launcher Drives

Front steering launchers with movable mirrors in the plasma ${ }^{64}$ vessel provide the desired injection angle for each gyrotron. Figure 1 shows a photograph of a front steering launcher. The mirrors are operated via push rods which serve at the same time as cooling pipes. The mapping from the drive positions to the mirror adjustment is a rather large algebraic expression. Figure 3 shows a graph of this mapping. A PLC algorithm makes sure that the mirror motion avoids the "forbidden" area in order to ${ }_{72}^{71}$ prevent the launcher from damage.

On the opposite wall of the launchers 130 waveguide probes are integrated in the graphite tiles (65 per MBWG). Figure $4^{74}$ shows a photograph taken during the assembly. These electron cyclotron absorption (ECA) probes will be used to measure the ${ }^{76}$ RF transmission through the plasma. This can be used max- ${ }^{77}$ imize the absorption with the help of the polarizers. This is ${ }^{78}$ important especially in the O2-regime at higher densities where the single-pass absorption can be as low as $50 \%$. Therefore the tiles serve also as reflectors for the spent beam in order to obtain

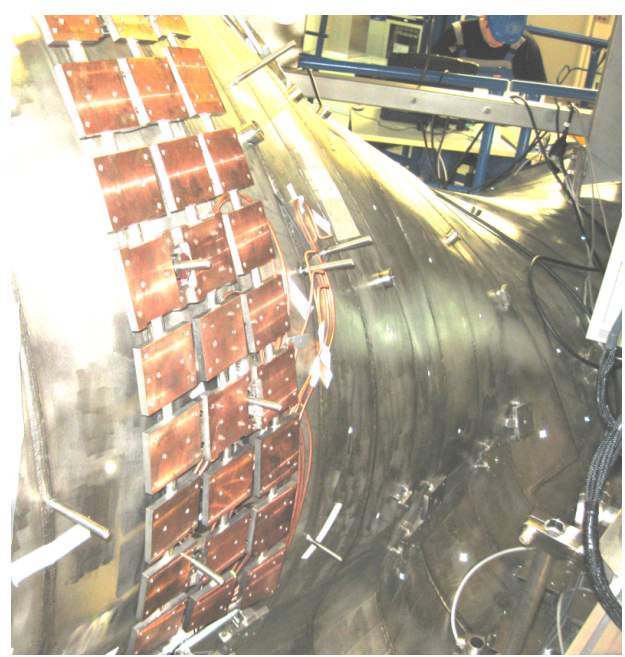

Figure 4: Tiles with ECA Probes at the Inner Wall

$$
7 \text { ers }
$$
mode composition of the beam. The polarizers are equipped with sinusoidal grooves in order to influence the wave polarization. These grooves are small compared to the transverse wave number and have therefore no influence on the beam except from the polarization. The sinusoidal shape of the surface diminishes the risk of arcing on the polarizer surface compared to rectangular grooves.

The first polarizer P1 provides the necessary amount of ellipticity to the originally linearly polarized beam. Polarizer P2 is used to rotate the output of $\mathrm{P} 1$. In the following, the procedure to find the proper adjustment angle of both polarizers will be discussed. 


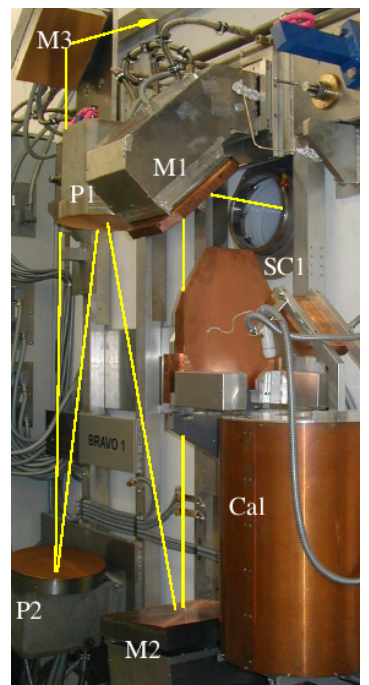

Figure 5: The Matching Optics Unit (MOU)

\section{Numerical Model}

The structures of the transmission line are very large compared to the wavelength $(2.1 \mathrm{~mm})$. Therefore, a full-wave calculation is numerically expensive. Usually, the field is decomposed into a set of modes which are propagated separately. At the target location the modes are added up again. Typically, Gauß-Hermite or Gauß-Laguerre modes are used. Unfortunately, there exists no fast decomposition algorithm for these modes. Therefore plane waves in conjunction with a two-di-131 mensional FFT are used. For the complete description of $a^{132}$ beam with a time dependency of $e^{+j \omega t}$ the Ansatz

$$
\mathbf{F}(x, y, 0)=u(x, y) \mathbf{F}_{0}
$$

is chosen. The plane $z=0$ describes an aperture in its local ${ }^{136}$ coordinate system. The complex function $u$ represents the field ${ }^{137}$ distribution or mode mixture in the aperture, and $\mathbf{F}_{0}$ is a con- ${ }^{138}$ stant three-dimensional complex vector of the electric vector ${ }^{139}$ potential which characterizes the polarization state. The electric vector potential $\mathbf{F}$ fulfills the Helmholtz equation and is defined by $\mathbf{E}=\nabla \times \mathbf{F} / \varepsilon_{0}$. The physical entities $\mathbf{E}$ and $\mathbf{H}$ can then be derived from $\mathbf{F}=u \mathbf{F}_{0}$. See [7] for a detailed description.

For the matching problem only the polarization state is of interest. Therefore the mode mixture is not important and it is legitimate to propagate only $\mathbf{F}_{0}$. This reduces the numerical effort drastically and allows for an instantaneous calculation of the (back-)propagation of a microwave beam.

$\mathbf{F}_{0}$ is altered at every mirror surface, especially at the polarizers. On plain mirrors, only the normal component $F_{z}$ changes its sign. This implies e.g. that the rotation sense of an elliptically polarized beam is inverted.

The transformation of $\mathbf{F}_{0}$ on the polarizers is more complicated. It is determined by the angle of the grooves with respect ${ }^{141}$ the plane of incidence and the geometry of the grooves. $\mathrm{An}^{142}$ analytical model for the transformation of the electric field for ${ }^{143}$ rectangular grooves has been developed by Kok and Gallagher ${ }^{144}$ [4]. In [5] it was shown that this model is also applicable for ${ }^{145}$ sinusoidal grooves if an "effective" groove depth is chosen. In order to fit to (1), the reflection on the polarizers is modeled by first calculating $\mathbf{E}_{0 \text {,inc. }}$ from $\mathbf{F}_{0 \text {,inc. }}$. Then $\mathbf{E}_{0 \text {,refl. }}$ is calculated by the Kok-Gallagher model and finally transformed into $\mathbf{F}_{0 \text {,refl. }}$ [7]. With this method the polarization state can be propagated from the gyrotron output to the plasma boundary.

For an ideal elliptical polarizer with a linearly polarized input beam (from the gyrotron) the orientation of the main axes of the polarization ellipse of the output beam follows the polarizer angle. The signed compression ratio changes linearly from -1 to 1 when the polarizer moves from $-45^{\circ}$ to $45^{\circ}$. Usually the compression ratio of an ellipse is the ratio of the smaller to the bigger main axis. The signed compression ratio in this case comprises also the rotation sense which is essential for the wave matching.
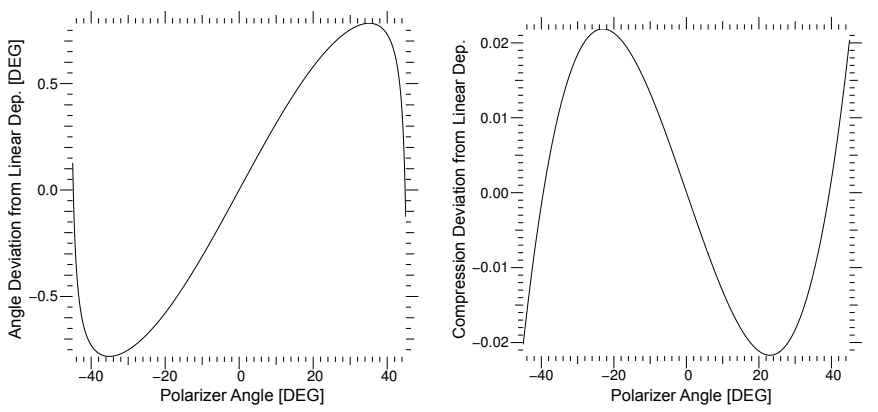

Figure 6: Deviation of the Elliptical Polarizer from Ideal Behavior

An analysis of the elliptical polarizer with the Kok-Gallagher model shows that its behavior is close to ideal, see Figure 6. The same is true for the linear polarizer. The compression ratio of its output should always be zero for a linearly polarized input. In this model it remains mostly below 0.03 . The deviation of the rotation angle from the ideal behavior is always smaller than $0.7^{\circ}$, see Figure 7 . Note that the rotation sense of the polarization ellipse does not change on this polarizer in contrast to plain mirrors. Because of the small errors ideal behavior for both polarizers is assumed in the following.
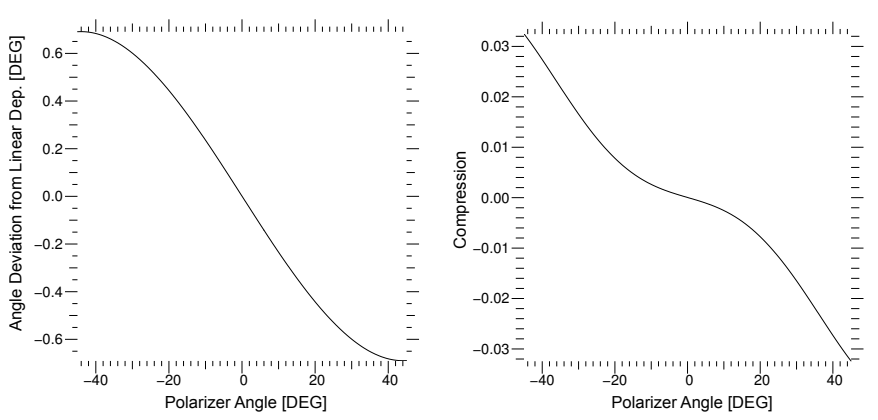

Figure 7: Deviation of the Linear Polarizer from Ideal Behavior

In order to match the microwave beam to the plasma boundary the task is now to find a mapping $\mathbb{R}^{2} \rightarrow \mathbb{R}^{2}$ from the launcher adjustment (toroidal and poloidal angle) to the polarizer angles. First, the polarization ellipse at the plasma boundary must be found. It depends on the local $\mathbf{B}_{0}$, which is obtained 
from the TRAVIS code [6], developed at IPP. This is a mature ${ }_{181}$ and sophisticated raytracing code for which the delivery of $\mathbf{B}_{0182}$ at the plasma boundary for a given injection angle is only a sec-183 ondary feature. Nevertheless, this code comes in handy here because of its good usability.

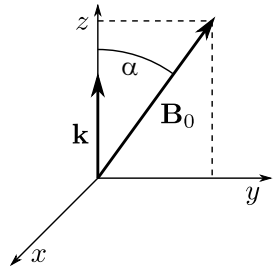

Figure 8: Local Coordinate System at the Plasma Boundary

The two vectors $\mathbf{B}_{0}$ and $\mathbf{k}$ at the plasma boundary determi the polarization state for $\mathrm{O}$ - and X-mode. A local coordinate ${ }_{193}$ system is obtained according to Figure 8 by defining the $\mathrm{X}$-axis ${ }_{194}$ as $\mathbf{B}_{\mathbf{0}} \times \mathbf{k} /\left(B_{0} k_{0}\right)$ and the $y$-axis accordingly. With the help of $\mathrm{a}_{195}$ good textbook (e.g. [3]) one can find the polarization state for ${ }_{196}$ $\mathrm{O}$ - and X-mode in this coordinate system after some simplifi- ${ }_{197}$ cation:

$$
\frac{F_{0, x}}{F_{0, y}}=j \frac{2 \cos \alpha}{\sqrt{s} \sin ^{2} \alpha \pm \sqrt{s \sin ^{4} \alpha+4 \cos ^{2} \alpha}}, F_{0, z}=0
$$

with $s=\omega_{c}^{2} / \omega^{2}$, where $\omega_{c}$ is the electron cyclotron frequency ${ }_{201}$ at $B_{0}$. The upper sign in the denominator gives the result for the ${ }_{202}$ $\mathrm{X}$-mode and the lower sign for the $\mathrm{O}$-mode. The simplification ${ }^{203}$ lies in the fact that a collisionless plasma with zero density is ${ }^{204}$ assumed, which is justified at the plasma boundary.

Equation (2) divided by $j$ delivers directly the sioned com 200 pression ratio $c$ of the polarization ellipse and hence, the ac-208 cording angle $\beta_{1}=c \cdot 45^{\circ}$ of the elliptical polarizer P1. Now the ${ }^{209}$ remaining task is to backpropagate the beam from the plasma ${ }_{211}^{210}$ to a reference plane right after the polarizers and to find the ori-212 entation of the main axes of the polarization ellipse there. This ${ }^{213}$ in turn allows to find the according angle of the linear polarizer ${ }^{214}$ $\mathrm{P} 2$. As $u$ in (1) is not taken into account, the backpropagation ${ }_{216}^{215}$ consists merely of the transformation of $\mathbf{F}_{0}$ on every mirror sur-217 face. For the front steering launchers we have 14 mirrors from ${ }^{218}$ the plasma to the reference plane.

In the reference plane, the transverse components of $\mathbf{F}_{0} \mathrm{de}_{-221}^{220}$ termine the orientation of the main axes of the polarization el-222 lipse. In a coordinate system where $\mathbf{k} \| \mathbf{e}_{z}$ one can find the main ${ }^{223}$ axes of the polarization ellipse in the $x-y-$ plane

$$
\mathbf{A}=\mathfrak{R}\left\{\begin{array}{l}
F_{0, x} e^{j \varphi} \\
F_{0, y} e^{j \varphi}
\end{array}\right\}
$$

where

$$
\begin{aligned}
\varphi & =\arctan \left(p \pm \sqrt{p^{2}+1}\right) \\
p & =\frac{\mathfrak{R}\left\{F_{0, x}^{2}\right\}+\mathfrak{R}\left\{F_{0, y}^{2}\right\}}{\mathfrak{J}\left\{F_{0, x}^{2}\right\}+\mathfrak{J}\left\{F_{0, y}^{2}\right\}} .
\end{aligned}
$$

The upper or the lower sign in (4) has to be chosen such that $\mathbf{A}$ is the larger of both main axes. Then the angle for polarizer P2 is

$$
\beta_{2}=\frac{\arctan \left(\mathbf{A}_{y} / \mathbf{A}_{x}\right)-\beta_{1}}{2} .
$$

This includes the correction of the rotated main axes from P1. Now the polarizer angles $\beta_{1}$ and $\beta_{2}$ produce $\mathrm{X}$ - or O-mode according to (2).

\section{Conclusions}

The above model can only be a starting point for the fine adjustment of the polarizers with the help of the ECA probes at the high field side of the plasma. This is due to the simplifications made above and to the error in the prediction of $\mathbf{B}_{0}$ and the location of the plasma boundary. Nevertheless, the matching should be good enough for the plasma start-up and to prevent from unacceptable amounts of stray radiation in the torus in the first operation phase of W7-X. The next step will be the production of a lookup-table to generate the launcher-polarizer mapping for the complete operating range of the launchers for both $\mathrm{O}$ - and $\mathrm{X}$-mode.

\section{References}

[1] V. Erckmann, P. Brand, H. Braune, G. Dammertz, G. Gantenbein, W. Kasparek, H.P. Laqua, H. Maaßberg, N.B. Marushchenko, G. Michel, M. Thumm, Y. Turkin, M. Weißgerber, A. Weller, Electron Cyclotron Heating for W7-X: Physics and Technology, Fusion Sci. Technol. 52, pp. 291-312, 2007

[2] M. Hirsch, J.W. Oosterbeek, R. Jimenez, J. Baldzuhn, M. Endler, M. Laux, D. Zhang, H.P. Laqua, F. Noke, F. Purps, K. Ewert and the W7-X Team, Qualifying Plasma Diagnostics for a high-power microwave background of ECR-heated diacharges, Proceedings of the International Conference on Plasma Diagnostics, April 12-16, 2010, Pont-à-Mousson, France, AIP conference proceedings

[3] V.L. Ginzburg, Propagation of Electromagnetic Waves in Plasma, Gordon and Breach, Science Publishers, Inc., 1961

[4] Y.-L. Kok, N.C. Gallagher, Relative phases of electromagnetic waves diffracted by a perfectly conducting rectangular-grooved grating, J. Opt. Soc. Am. A, vol. 5, No. 1, pp. 65-73, 1988

[5] D. Wagner, F. Leuterer, Broadband Polarizers for High-Power MultiFrequency ECRH Systems, Int. J. of IRMM Waves, vol. 26, No. 2, pp. $163 \mathrm{ff}, 2005$

[6] N.B. Marushchenko, H. Maßßberg, Yu. Turkin, Electron cyclotron current drive calculated for ITER conditions using different models, Nucl. Fusion 48, 054002, 2008

[7] G. Michel, Numerical Modeling of Millimeter-Wave Transmission Lines, IEEE Trans. Plasma Sci., vol. 38, No. 6, pp. 1375-1384, 2010 